\title{
Use of soft x-ray diagnostic on the COMPASS tokamak for investigations of sawteeth crash neighborhood and of plasma position using fast inversion methods
}

\author{
M. Imrisek ${ }^{1,2}$, V. WeinzettI ${ }^{1}$, J. Mlynar ${ }^{1}$, T. Odstrcil ${ }^{3}$, M. Odstrcil ${ }^{4,5}, \mathrm{O}$. \\ Ficker $^{4}$, J.R. Pinzon ${ }^{6}$, C. Ehrlacher ${ }^{7}$, R. Panek ${ }^{1}$ and M. Hron ${ }^{1}$ \\ ${ }^{1}$ Institute of Plasma Physics ASCR, Prague, Czech Republic \\ ${ }^{2}$ Charles University in Prague, Faculty of Mathematics and Physics, Prague, Czech Republic \\ ${ }^{3}$ Max-Planck-Institut fürPlasmaphysik, Garching, Germany \\ ${ }^{4}$ Czech Technical University in Prague, Faculty of Nuclear Sciences and Physical Engineering, Prague, \\ Czech Republic \\ ${ }^{5}$ University of Southampton, Southampton, United Kingdom \\ ${ }^{6}$ Université de Lorraine, Institue Jean Lamour, Nancy, France \\ ${ }^{7}$ ENS Cachan, Paris, France
}

\begin{abstract}
(Presented XXXXX; receivedXXXXX; accepted XXXXX; published online XXXXX)
The soft $X$-ray diagnostic is suitable for monitoring plasma activity in the tokamak core, e.g. sawtooth instability. Moreover, spatially resolved measurements can provide information about plasma position and shape, which can supplement magnetic measurements. In this contribution, fast algorithms with the potential for a real-time use are tested on the data from the COMPASS tokamak. In addition, the soft x-ray data are compared with data from other diagnostics in order to discuss possible connection between sawtooth instability on one side and the transition to higher confinement mode, Edge localized modes and runaway electrons on the other side.
\end{abstract}

\section{SOFT X-RAY DIAGNOSTIC ON THE COMPASS TOKAMAK}

In tokamak plasmas without heavy impurities, the soft x-ray (SXR) radiation is generated mainly by bremsstrahlung which depends on both electron density $n_{e}$ and temperature $T_{e}$ :

$$
P_{\text {brem }} \sim Z_{\text {eff }} n_{e}^{2} \sqrt{T_{e}}
$$

where $Z_{\text {eff }}=\sum_{i} n_{i} Z_{i}^{2} / n_{e}$ is effective ion charge, and $n_{i}$ is density of $Z_{i}$-times charged ions. Since the temperature profile is usually peaked in the tokamak plasma core and density can have flat as well as peaked profile, the SXR diagnostic is suitable for monitoring plasma core activity, e.g. the sawtooth instability. Moreover, spatially resolved measurements can provide information about plasma position and shape. Measured signals always correspond to chord integrated soft x-ray radiation, therefore, inversion methods (tomography), which are reviewed in section II, have to be applied in order to obtain spatial distribution of the plasma emissivity.

The COMPASS tokamak, a divertor device with clear $\mathrm{H}$ mode and ITER-relevant geometry (1:10 to ITER plasma size, $\mathrm{R}=0.56 \mathrm{~m}, \mathrm{a}=0.23 \mathrm{~m}, \mathrm{I}_{\mathrm{p}}<400 \mathrm{kA}, \mathrm{B}_{\mathrm{T}} \sim 1.15 \mathrm{~T}$ and typical pulse length around $300 \mathrm{~ms})^{1}$ is equipped with the fast SXR diagnostic system $^{2}$ based on silicon photodiode arrays shielded by the beryllium foil of thickness of $10 \mu \mathrm{m}$. The diagnostic is composed of two pinhole cameras having 35 channels each (Centronic, type LD35-5T) and one vertical pinhole camera with 20 channels which has been installed recently (IRD, type AXUV-20EL) (see FIG. 1) to improve tomographic inversions. Spatial resolution of each camera is about $1-2 \mathrm{~cm}$. Temporal resolution of the SXR

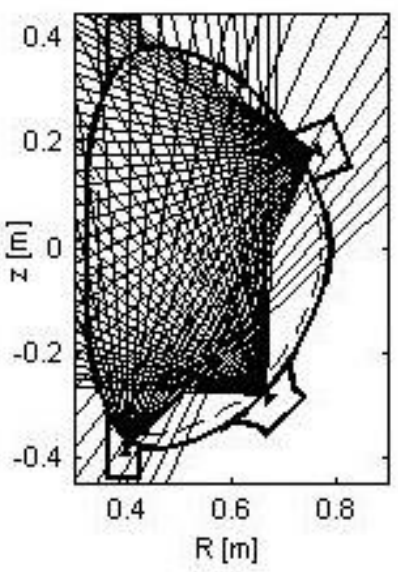
diagnostic is constrained mainly by the amplifier to about $3 \mu \mathrm{s}$ allowing an investigation of fast $\mathrm{MHD}$ processes and instabilities in the plasma core. The spectral sensitivity of the system is mainly determined by thickness of beryllium foil $(10 \mu \mathrm{s})$ filtering out photons with energy below $1 \mathrm{keV}$ and by thickness of diode active

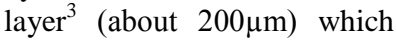
limits the detector sensitivity to photon energies below $13 \mathrm{keV}$.

FIG. 1.Geometry of SXR detectors on the COMPASS tokamak

\section{INVERSION METHODS}

Local emissivity can be estimated by inversion methods for a discrete set of rectangular pixels, so that the relationship between line integrated data $f_{i}$ and emissivity distribution $g_{i}$ can be written as ${ }^{3}$

$$
f_{i}=\sum_{j}^{N} T_{i j} g_{j}+\zeta_{i}
$$


where $T_{i j}$ is the contribution matrix, $N$ number of pixels and $\zeta_{i}$ statistical errors. However, tokamak plasma projections are sparse due to the engineering constraints and, moreover, the inversion represents an ill-conditioned task. In order to find a unique solution, regularization algorithms constrained by a priori information (e.g. expected smoothness) are used. A unique solution can be found by minimizing:

$$
\Lambda=\frac{1}{2} \chi^{2}+\alpha F(g)
$$

where $\chi^{2}$ is the goodness-of-fit parameter, $\alpha$ is the regularization (smoothing) parameter and $F$ is the regularization functional. Minimum Fisher regularization ${ }^{4}$ (MFR), is often applied to find a smooth solution by choosing the Fisher information as the regularization functional. MFR has proved to find robust and reliable solution ${ }^{5}$. Weighted anisotropic derivatives with respect to the magnetic flux surfaces in the Fisher information allow finding a solution with higher smoothness along magnetic field lines.

Among other parameters, tomographic reconstruction can be used to evaluate the center of mass of the SXR emission related to the plasma position. Therefore, the center of mass of the SXR radiation could be used to supplement magnetic measurements of plasma position, which may suffer from integration drifts during long shots. However, standard tomography reconstruction of plasma profile is inconvenient for real-time calculations. Simplified linear methods allow faster reconstruction in exchange for lower precision. Their main advantage is the possibility to precalculate a decomposition of the emissivity distribution into global basis functions (depending only on the geometry of experiment). Then, it can be used for calculation of weight vectors for distribution moments. In the linear methods used in this contribution, the regularization is constrained by the smoothness of solution, i.e. $F(g)=\|\mathbf{C} g\|^{2}$, where $\mathbf{C}$ is numerical differentiation matrix. During the minimization of eq. (4), the decomposition $\mathbf{T C}^{-1}=\mathbf{U} \boldsymbol{\Sigma} \mathbf{V}^{T}$ by $\operatorname{SVD}^{6}$ (Singular Value Decomposition), $\mathrm{QR}^{7}$ based method or $\mathrm{GEV}^{8}$ (Generalized Eigen Values) based method leads after several steps to a solution in the form:

$$
g(\alpha)=\sum_{j} w_{j}(\alpha) \frac{f \cdot u_{j}}{\sigma_{j}} C^{-1} v_{j}
$$

where $u_{j}$ and $v_{j}$ are columns of $\mathrm{U}$ and $\mathrm{V}, \sigma_{j}$ diagonal elements of singular values $\Sigma$ and $w_{j}(\alpha)=\left(1+\alpha / \sigma_{j}^{2}\right)^{-1}$ can be seen as low-pass filter (cuts off components with higher $j$ as $\sigma_{j}$ decreases). It is observed, that the optimal value of $\alpha$ can be chosen as $\operatorname{med}(\Sigma)$. This approach is independent on measured data (in contrast to the generalized cross-validation ${ }^{5}$ or finding the roots of $\chi^{2}=1$ ). The center of mass of SXR radiation can be then derived from eq. (5) as scalar multiplication of measured data with precomputed weight vectors for the first moments $b_{1 r}$, $b_{1 z}$ normalized by scalar multiplication of measured data with the zero'th moment $b_{0}$ :

$$
\left[r_{c t r}, z_{c t r}\right]=\left[\frac{b_{1 r} \cdot f}{b_{0} \cdot f}, \frac{b_{1 z} \cdot f}{b_{0} \cdot f}\right]
$$

Furthermore, higher moments providing information about plasma shape can be obtained in a similar way. Scalar multiplication of measured data by weight vectors is faster (several $\mu \mathrm{s}$ ) than sophisticated tomographic methods as MFR (hundreds of ms) which is (beside the precision) a crucial advantage for the real-time plasma position control. Weight vectors calculated by different matrix decomposition techniques as SVD, QR and GEV are used to obtain the center of mass of SXR radiation. The results are comparable with the MFR-based tomography (see FIG. 2). The GEV method provides results with lower standard deviations of difference between magnetic and SXR-based positions $(1.2 \mathrm{~cm}$ for horizontal and $1.6 \mathrm{~cm}$ for vertical direction) than the QR and the SVD method. This root-meansquare-deviation is comparable to the spatial resolution of the SXR detectors. The average horizontal SXR positions are positioned closer to the high field side as a result of Shafranov shift and SXR profile is affected by plasma configuration.
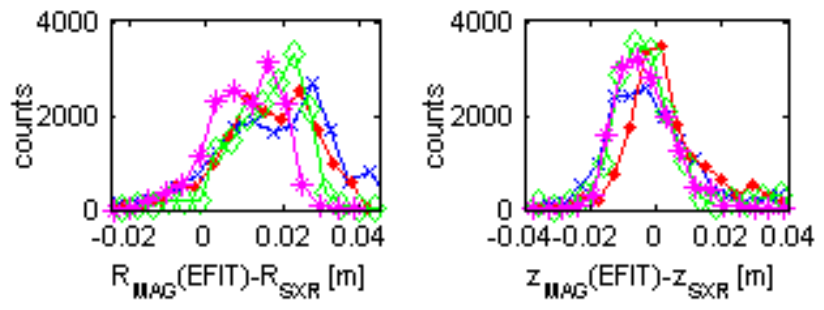

FIG2.(Color online). Histogram of horizontal(right) and vertical (left) differences between magnetic axis (reconstructed by EFIT) and the center of mass of SXR radiation obtained by full tomographic reconstruction (MFR denoted by magneta stars) comparing fast methods based on precomputing weight vectors using SVD (blue x-marks), QR (red dots), GEV (green diamonds). Data were obtained from 90 various discharges with a typical D-shaped configuration.

\section{STUDIES OF SAWTOOTH INSTABILITY}

The sawtooth oscillation is one of the fundamental instabilities occurring in the plasma core. Repetitive slow increase and fast drop in both the core temperature and density can be seen as sawtooth pattern on several signals, including in particular $\mathrm{SXR}^{9}$. The sawtooth instability is an important topic in the fusion research, because it affects the hottest part of the plasma volume, and triggers other instabilities below their threshold ${ }^{9}$. On the other hand, it can help to remove helium ash from the plasma core. The sawtooth crash is usually accompanied by the internal kink mode $m=n=1$ which can be seen as precursor oscillations before the sawtooth crash. Kadomtsev mode ${ }^{10}$ has provided a starting point for an understanding of the sawtooth crash, however, it is in contradiction with several measurements ${ }^{11,12}$ Presently, a partial reconnection model ${ }^{13}$ is often preferred for the interpretation of the sawtooth crash. For example, oscillations of the hot core in reconstructed radial profile (see FIG. 3) show that the mode position remains unchanged after the crash which is in contradiction with the

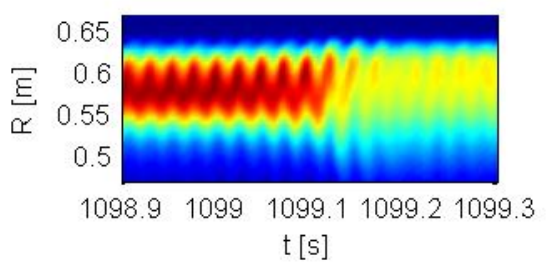

Kadomtsev model. However, absence of the post-crash oscillations violating the partial reconnection model is also observed.

FIG 3.(Color online). A reconstructed radial profile of SXR emissivity during the internal kink mode accompanying the sawtooth crash at $1099.12 \mathrm{~ms}$. 
The sawtooth crash can trigger the L-H transition ${ }^{14}$. It is supposed that the hot plasma expelled from the central region to the edge can supply enough free energy to the formation of the edge transport barrier. The synchronization of the L-H transition with the sawtooth crash is very clear at the COMPASS tokamak as it can be seen in the FIG. 4 left. In contrast to this observation, there is no clear evidence of synchronization of the sawtooth crash with the H-L transition (see FIG. 4 right).
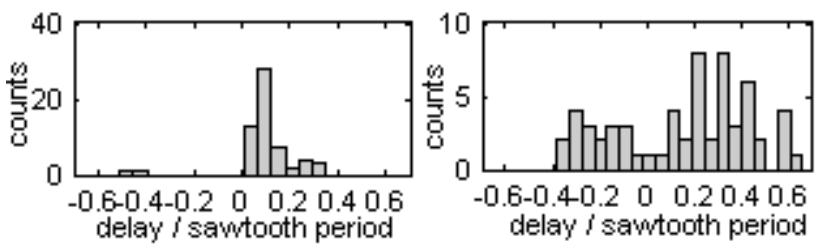

FIG. 4.The delay of the L-H (left) and H-L (right) transition after the sawtooth crash.

During the sawtooth crash, hard X-ray radiation (HXR) has been often detected on COMPASS (see FIG. 5). HXR is usually observed in low density plasmas and during disruptions as a consequence of runaway electrons generation due to low collisionality and strong toroidal electric field. A possible mechanism of the electron acceleration during the sawtooth crash proposed by Klimanov ${ }^{15}$ suggests that runaways are driven by magnetic reconnection. However, no significant relation between the amplitude of sawteeth and HXR intensity caused by runaways was observed on the COMPASS tokamak.

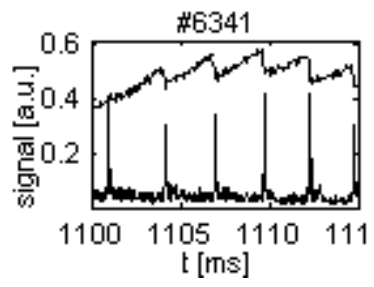

FIG. 5. Typical SXR signals (upper line) during the sawtooth instability and measured hard $\mathrm{x}$-ray (HXR) radiation (lower line). The HXR peaks indicate interaction between runaway electrons and the vessel.

On rare occasions, sawtooth crashes are observed to tightly precede edge localized modes (ELM), see FIG. 6 left. Although Nave ${ }^{16}$ observed larger sawteeth crashes coupling with ELMs, on COMPASS not focusing at any particular plasma parameter, synchronisation of the ELMs and sawtooth crashes is not related to the amplitude of the sawtooth instability. A possible correlation between ELM and sawtooth instabilities is indicated by a drop in ELM distribution about $0.5 \mathrm{~ms}$ after the sawtooth crash. However, more extensive statistics is necessary for proper evaluation of this effect.
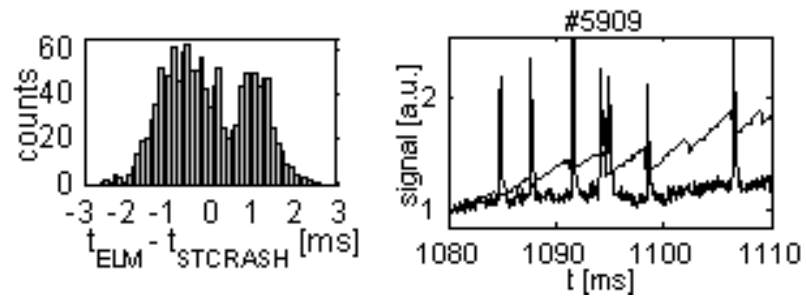

FIG. 6. ELM distribution with respect to the time of the sawtooth crash (left) and observed coupling of ELMs with sawtooth crashes (right).

\section{DISCUSSION AND SUMMARY}

Fast algorithms for calculation of the center of SXR radiation center have been successfully applied on data from the COMPASS tokamak. In further applications, it is to be noted that the center of mass of SXR radiation can be affected by asymmetry in density profile of the heavy impurities (in particular in tokamaks with tungsten divertor), which would require corrections for centrifugal force and other influences. Information about the poloidal asymmetry can be partly provided by higher moments. Nevertheless, the linear methods allow fast calculation of the distribution moments which are more convenient for real-time use. Limitations, possible corrections and a potential of fast algorithms for real-time use are to be further examined. Furthermore, the SXR data were used to investigate the plasma core activity during the sawtooth crash and also the SXR emissivity was compared with other diagnostics to show clear triggering of the L-H transition by the sawtooth crash and to discuss the influence of the sawtooth instability on the ELMs and on the runaway electrons. More data and further studies, e.g. the role of inversion radius in the sawteeth instability as obtained from SXR tomography ${ }^{3}$ and/or the amplitude of instability could further clarify possible influence of the sawtooth instability on the L-H transition, ELMs and runaway electrons.

\section{AKNOWLEDGEMENTS}

This work was supported by grant of the Czech Science Foundation GA P205/10/2055 as well as by the MSMT projects LM2011021.

${ }^{1}$ Pánek R, Bilyková P, Fuchs V, Hron M, Chráska P, Pavlo P, Stockel J, Urban J, Weinzettl V, Zajac J and Zacek F: Czechoslovak J. Phys. 2006 56/2 B125-137

${ }^{2}$ V.Weinzettl, D.I.Naydenkova, D.Sestak, J.Vlcek, J.Mlynar, R.Melich, D.Jares, J.Malot, D.Sarychev, V.Igochine, Nucl. Instr. and Methods in Phys. Res. A 623 (2010) 806-808

${ }^{3}$ V. Igochine, A. Gude, M. Maraschek and ASDEX Upgrade team, Internal IPP report 1/338 (2010)

${ }^{4}$ M. Anton, H.Weisen, M. J. Dutch,W. Linden, F. Buhlmann, R. Chavan, B.Marletaz, P. Marmillod, and P. Paris, Plasma Phys. Controlled Fusion 38,1849 (1996)

${ }^{5}$ J. Mlynar, M. Imrisek, V. Weinzettl, M. Odstrcil, J. Havlicek et al.Rev. Sci. Instrum. 83, 10E531 (2012)

${ }^{6} \mathrm{~N}$. Terasaki, Y. Hosoda, M. Teranishi and N. Iwama, Fusion engineering and design, 34-35 (1997) 801-804

${ }^{7}$ Y. Hosoda and T. Torii, Jpn. J. appl. Mat, pages 287-298 (1994)

${ }^{8}$ G. C. Fehmersy, L. P. J. Kamp and F. W. Sluijter, Inverse Problems 14 (1998) 893-901

${ }^{9}$ I. T. Chapman IT et al, Plasma Phys. Control. Fusion 53013001 (2011)

${ }^{10}$ B. B. Kadomtsev, Sov. J. Plasma Phys. 1389 (1976)

${ }^{11}$ J. Blum, E. Lazzaro, J. O'Rourke, B. Keegan and Y. Stephan, Nucl. Fusion 301475 (1990)

${ }^{12}$ A. W. Edwards et al, Phys. Rev. Lett. 57210 (1986)

${ }^{13}$ F. Porcelli, D. Boucher and M. Rosenbluth Plasma Phys. Control. Fusion 382163 (1996)

${ }^{14}$ Y. R. Martin and TCV team, Plasma Phys. Controlled Fusion 46, A77A85 (2004)

${ }^{15}$ I. Klimanov, A. Fasoli, T. P. Goodman and the TCV team, Plasma Phys. Control. Fusion 49, L1-L6 (2007)

${ }^{16}$ M.F.F. Nave et al, Nucl. Fusion 35509 (1995) 